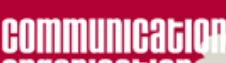
Oryanllatín.
Communication et organisation

Revue scientifique francophone en Communication organisationnelle

$60 \mid 2021$

Influence et organisations : cultures, pratiques et mises en perspective

\title{
VIALLON Philippe et GARDÈRE Elizabeth. Médias dits sociaux ou médias dissociants?
}

Bruxelles : De Boeck supérieur, 2020

\section{Nadia Hassani}

\section{CpenEdition}

\section{Journals}

Édition électronique

URL : https://journals.openedition.org/communicationorganisation/10839

DOI : 10.4000/communicationorganisation.10839

ISSN : 1775-3546

Éditeur

Presses universitaires de Bordeaux

Édition imprimée

Date de publication : 1 décembre 2021

Pagination : 191-193

ISSN : 1168-5549

Référence électronique

Nadia Hassani, «VIALLON Philippe et GARDÈRE Elizabeth. Médias dits sociaux ou médias

dissociants ? », Communication et organisation [En ligne], 60 | 2021, mis en ligne le 10 janvier 2022

consulté le 15 juin 2022. URL : http://journals.openedition.org/communicationorganisation/10839

DOI : https://doi.org/10.4000/communicationorganisation.10839 


\title{
Médias dits sociaux ou médias dissociants? VIALLON Philippe et GARDÈRE Elizabeth, 2020.
}

\author{
Auteure de la recension : Nadia HASSANI
}

Cet ouvrage est paru aux éditions De Boeck Supérieur (Bruxelles) en novembre 2020. Il est écrit par deux professeurs des Universités en sciences de l'information et de la communication. En 9 chapitres, les auteurs offrent une mise en perspective particulièrement éclairante des " médias sociaux " qui regroupent à la fois "les plateformes, mais aussi les forums et blogs ainsi que les sites Web» (p. 12).

L'introduction et le premier chapitre de ce livre proposent un rappel théorique des notions fondamentales en matière de communication numérique, offrant ainsi au lecteur la possibilité de découvrir les origines et les évolutions du concept de " média social » et de le situer dans un contexte plus global.

Le deuxième chapitre intitulé "L'écosystème des réseaux sociaux " est dédié à l'histoire des théories des sciences de l'information et de la communication. Se référant notamment à l'école de Palo Alto, les auteurs mettent en évidence la dimension relationnelle et interactionniste des médias sociaux, démontrant que l'utilisation des réseaux sociaux numériques n'est autre qu’un processus médiatisé à travers lequel nous construisons notre propre réalité.

Le chapitre suivant " réseaux sociaux et écologie environnementale " met en lumière l'empreinte écologique liée à une utilisation massive et globalisée des médias sociaux. Le concept de responsabilité, collective et individuelle, est ici engagée.

Le quatrième chapitre invite le lecteur à interroger l'impact des médias sociaux sur notre rapport à la temporalité ("fragmentée ", "instantanée ", " désynchronisée ", p. 55-58) et à l'espace (" éclaté », " disloqué ", " recomposé », p. 60-61). Les notions de virtualité (p.64), d'incommunication et de fake news (p. 65), d'identité numérique (p. 67), ou encore d'individualisme et de culture participative (p. 69) y sont explorées avec une fluidité remarquable.

"L'économie des médias sociaux ", cinquième chapitre de l'ouvrage, propose un panorama "non exhaustif » de ce pan de l'économie du numérique. Les auteurs y abordent l'économie du partage, du clic, de la donnée, de la confiance ou de la connaissance, toutes intrinsèquement liées aux modèles économiques des réseaux sociaux numériques.

Alors que le sixième chapitre questionne la règlementation actuelle et les usages dérégulés des médias sociaux à l'heure des "pratiques addictives " du numérique (p. 95), le septième chapitre aborde la citoyenneté et la régulation des médias dits " sociaux ». Constatant l'échec des régulations sociale et citoyenne, les auteurs 
convoquent à nouveau l'approche systémique qui permettrait de limiter « les lois complexes " et le " cybercontrôle " du fait qu' elle "permet de comprendre en quoi une dysfonction peut servir la régulation» (p. 113).

Le huitième chapitre de cet ouvrage est consacré au monde de l'entreprise, où les réseaux sociaux sont, selon les auteurs, une opportunité pour instaurer un mode de management plus collaboratif (p. 116) et plus transversal (p. 121) qui fluidifie la circulation de l'information et optimise l'efficacité des collaborateurs (p. 127). Le neuvième et dernier chapitre propose un inventaire des nouveaux métiers liés à l'essor des médias sociaux dans les secteurs de l'informatique, de l'informationdocumentation, de la communication, du marketing et de la formation.

Dans leur conclusion, Philippe Viallon et Elizabeth Gardère questionnent la pérennité de ce modèle économique censé être basé sur la confiance alors qu'il n’a d'autre objectif que la "collecte de données à des fins mercantiles " (p. 147). Ils pointent également du doigt le risque de délitement du lien social (p. 147) ou d'atteinte à la liberté d'expression (p. 149). Les auteurs y soulignent enfin la dimension relationnelle, conversationnelle, communautaire et interactionnelle des médias dits sociaux, quand les réseaux sociaux "s'emparent de ce dialogue " pour ne s'adresser qu'aux individus à des fins publicitaires (p. 152).

Présenté par l'éditeur comme un ouvrage à destination des étudiants de Master ou de Doctorat, l'une des principales forces de cet ouvrage d'une grande clarté tient au fait qu'il s'adresse aussi bien à un public étudiant, enseignant ou professionnel. D'une part, les étudiants de tous niveaux y trouveront de nombreuses références théoriques et épistémologiques, étayées par une bibliographie et webographie particulièrement riche. D'autre part, les enseignants y verront un support pédagogique complet pour enrichir leurs enseignements tout en sensibilisant leurs étudiants aux enjeux actuels dus à l'essor des réseaux sociaux numériques. Enfin, les professionnels de la communication pourront y interroger leurs pratiques numériques et trouver des réponses pour les faire évoluer vers des modèles de management et de partage de l'information plus vertueux.

Référence complète : VIALLON Philippe et GARDÈRE Elizabeth, 2020. Médias dits sociaux ou médias dissociants ? Bruxelles : De Boeck supérieur. 978-2-8073-2932$4,24,5 €$.

Nadia HASSANI est docteure en sciences de l'information et de la communication et qualifiée aux fonctions de Maître de conférences dans la 71e section du CNU. Membre du laboratoire LERASS-CERIC, ses travaux de recherche portent sur la communication des organisations, la conduite du changement, les pratiques numériques professionnelles (médias sociaux, plateformes numériques, stratégies d'influence ou encore Big Data) ainsi que l'évaluation du bonheur au travail ou encore le phénomène d'hyperconnexion. 